\title{
STM OBSERVED SURFACE STRUCTURES AND MAGNETIC PROPERTIES OF MBE-GROWN METALLIC THIN FILMS
}

\author{
R. KALINoWSKI ${ }^{a}$, L.T. BACZEWSKI ${ }^{a}$, A. WAWRO $^{a}$, C. MEYER $^{b}$ \\ AND J. RAUEUSZKIEWICZ ${ }^{a}$ \\ ${ }^{a}$ Institute of Physics, Polish Academy of Sciences \\ Al. Lotników 32/46, 02-668 Warsaw, Poland \\ ${ }^{b}$ Laboratoire de Magnétisme L. Néel, CNRS, 38042 Grenoble Cedex 9, France
}

\begin{abstract}
Rare-earth epitaxial thin films of $\mathrm{Tb}$ and $\mathrm{Gd}$ of the thicknesses between $2 \mathrm{~nm}$ and $16 \mathrm{~nm}$ were deposited by means of molecular beam epitaxy method. The roughness of the rare-earth films measured by scanning tunneling microscopy was found to be in the range of $1-4.5 \mathrm{~nm}$. The influence of the roughness on the dipolar anisotropy and magnetocrystalline surface anisotropy was estimated. The magnetic measurements have shown that the Gd layers deposited on the Y buffer layers had an easy plane anisotropy. However, for $2 \mathrm{~nm}$ thick Gd layer deposited on $\mathrm{W}$ buffer layer the perpendicular anisotropy was observed. According to the roughness analysis the possible sources of the perpendicular anisotropy in this sample is mainly the magnetoelastic anisotropy, but the presence of the magnetocrystalline surface anisotropy also cannot be neglected.
\end{abstract}

PACS numbers: $75.70 . \mathrm{Ak}, 68.55 .-\mathrm{a}, 68.60 . \mathrm{Dv}$

\section{Introduction}

The quality of a surface plays an important role in the magnetic properties of metallic thin films. The magnetization orientation in thin films is determined by a competition between different magnetic anisotropy energies, i.e. magnetocrystalline anisotropy, strain induced magnetoelastic anisotropy, dipolar anisotropy, and thickness dependent surface anisotropy [1,2]. The last two anisotropies strongly depend on the film roughness [3]. The dipolar anisotropy, favoring the in-plane magnetization in an ideal flat film, in a real film consisting of an array of islands can shift the magnetization to the perpendicular direction $[3,4]$. The magnetocrystalline surface anisotropy resulting from the asymmetric

*e-mail: kalin@ifpan.edu.pl 
environment of surface atoms may lead to the perpendicular magnetization orientation. For the rough films this effect is reduced due to the lower asymmetry at the edges of the terraces [3]. The knowledge of the roughness parameters, i.e. the islands height and diameter, is needed to estimate which effect is dominant in the studied samples. In this work we have investigated the Tb and Gd epitaxial thin films deposited on the tungsten or yttrium buffer layers. The roughness measurements and the magnetic properties of deposited films in dependence of the magnetic layers thicknesses and used buffer layers will be discussed.

\section{Experimental details}

The $\mathrm{Tb}$ and Gd rare-earth (RE) epitaxial thin films were deposited by means of MBE method under the UHV conditions $\left(10^{-10} \mathrm{Tr}\right)$. The crystal structure and chemical purity of the films were checked in situ using reflection high energy electron diffraction (RHEED) and Auger electron spectroscopy (AES) respectively. The films were grown on the commercially a vailable sapphire $(11 \overline{2} 0)$ substrates in two types of structures:

$$
\begin{aligned}
& \text { type } \mathrm{A}: \mathrm{Al}_{2} \mathrm{O}_{3}(11 \overline{2} 0) / \mathrm{W}(110) / \mathrm{RE}(0001) / \mathrm{W}(110) / \mathrm{Au}, \\
& \text { type } \mathrm{B}: \mathrm{Al}_{2} \mathrm{O}_{3}(11 \overline{2} 0) / \mathrm{W}(110) / \mathrm{Y}(0001) / \mathrm{RE}(0001) / \mathrm{Y}(0001) / \mathrm{Au} .
\end{aligned}
$$

All layers, except the first $\mathrm{W}$ buffer layer where $T_{\text {substrate }}=1100^{\circ} \mathrm{C}$, were deposited at room temperature. The thicknesses of buffer and cover layers were as follows: $W_{\text {buffer }}-30 \mathrm{~nm}, Y_{\text {buffer }}-10 \mathrm{~nm}, W_{\text {cover }}$ and $Y_{\text {cover }}-10 \mathrm{~nm}, A u_{\text {cover }}$ $-15 \mathrm{~nm}$. The thicknesses of $\mathrm{Tb}$ and Gd layers were in the range of 2-16 nm. The scanning tunneling microscopy (STM) measurements were performed ex situ in constant current mode using the STM unit with chemically etched tungsten tips. The magnetization loops were measured by means of vibrating sample magnetometer (VSM) at $10 \mathrm{~K}$ temperature in parallel and perpendicular direction of the applied magnetic field.

\section{Results and discussion}

The RHEED measurements showed a very good epitaxial growth of $\mathrm{Tb}$ and Gd on both types of buffer layers - the observed RHEED streaks were sharp and long which suggested the $2 \mathrm{D}$ growth of RE layers. On the other hand, it should be noticed that the correlation length for RHEED electrons is of an order of $20 \mathrm{~nm}$, so the layers with islands of larger diameter would be also recognised by RHEED as a flat film.

The films roughness measured by STM was characterized using two parameters, $\Delta h$ - average height and $\Delta l$ - average diameter of islands observed in the STM images. As the STM measurements were performed ex situ, it was necessary to estimate the resulting total roughness added by buffer and cover layers. The roughness of buffer layers was controlled in the samples $\mathrm{Al}_{2} \mathrm{O}_{3} / \mathrm{W} 30 \mathrm{~nm}$ and $\mathrm{Al}_{2} \mathrm{O}_{3} / \mathrm{W} 30 \mathrm{~nm} / \mathrm{Y} 10 \mathrm{~nm}$ and was found to be $\Delta h=0.9-1.1 \mathrm{~nm}$ and $\Delta l=$ 20-30 nm in both cases. To obtain the total roughness of the buffer and cover layers we have measured the $A$ and $B$ type samples without the RE layers (calibration samples) and $\Delta h=2 \mathrm{~nm}$ and $\Delta l=33 \mathrm{~nm}$ were found. The RE roughness was obtained by subtraction of the calibration samples roughness from the total 
roughness of studied films. The results are presented in Table and the examples of STM images are shown in Fig. 1a and Fig. 2a.

TABLE

The roughness of $R E$ layers of different thichnesses deposited on $W$ and $W / Y$ buffer layers. (A), (B) means sample of type $A(W / R E / W / A u)$ or sample of type $B$ $(\mathrm{W} / \mathrm{Y} / \mathrm{RE} / \mathrm{Y} / \mathrm{Au})$.

\begin{tabular}{c|c|c|c|c|c|c|c|c}
\hline \hline & $\mathrm{r} 4(\mathrm{~A})$ & $\mathrm{r} 3(\mathrm{~B})$ & $\mathrm{r} 7(\mathrm{~A})$ & $\mathrm{r} 9(\mathrm{~B})$ & $\mathrm{r} 11(\mathrm{~A})$ & $\mathrm{r} 13(\mathrm{~B})$ & $\mathrm{r} 19(\mathrm{~A})$ & $\mathrm{r} 6(\mathrm{~B})$ \\
\hline $\mathrm{Tb}$ & - & - & - & - & $4 \mathrm{~nm}$ & $4 \mathrm{~nm}$ & $16 \mathrm{~nm}$ & $16 \mathrm{~nm}$ \\
$\mathrm{Gd}$ & $2 \mathrm{~nm}$ & $2 \mathrm{~nm}$ & $4 \mathrm{~nm}$ & $4 \mathrm{~nm}$ & - & - & - & - \\
$\Delta h$ & $1 \mathrm{~nm}$ & $1 \mathrm{~nm}$ & $1.6 \mathrm{~nm}$ & $1.7 \mathrm{~nm}$ & $1.7 \mathrm{~nm}$ & $1.6 \mathrm{~nm}$ & $4.5 \mathrm{~nm}$ & $4 \mathrm{~nm}$ \\
$\Delta l$ & $40 \mathrm{~nm}$ & $45 \mathrm{~nm}$ & $40 \mathrm{~nm}$ & $60 \mathrm{~nm}$ & $40 \mathrm{~nm}$ & $60 \mathrm{~nm}$ & $50 \mathrm{~nm}$ & $85 \mathrm{~nm}$
\end{tabular}
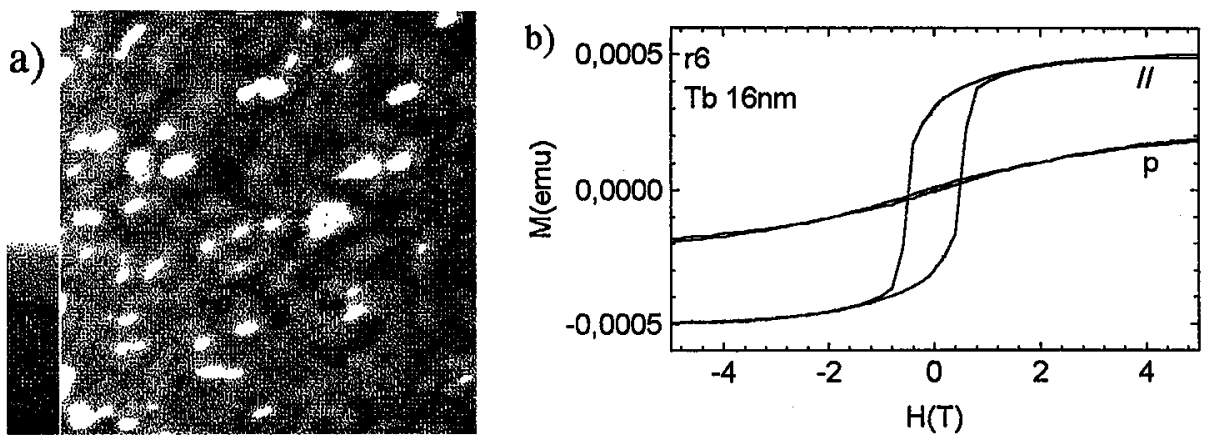

Fig. 1. (a) The STM image of the sample $\mathrm{r} 6$ (the image area cover $1 \times 1 \mu \mathrm{m}^{2}$, the height scale represents the range of $0-14 \mathrm{~nm}$ ); (b) the parallel (II) and perpendicular (p) magnetization loops of the sample $r 6$.
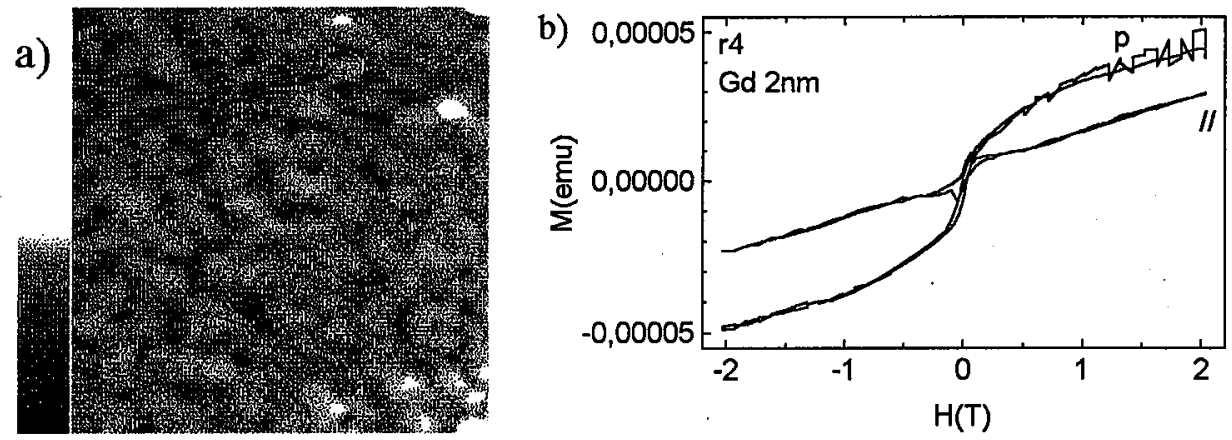

Fig. 2. (a) The STM image of the sample $\mathrm{r} 4$ (the image area cover $1 \times 1 \mu \mathrm{m}^{2}$, the height scale represents the range of $0-8 \mathrm{~nm})$; (b) the parallel $(\|)$ and perpendicular $(p)$ magnetization loops of the sample $r 4$. 
From the Table it is clear that the roughness of RE layers deposited at room temperature increases with the thickness of the film and does not depend on the type of buffer layer. The lateral size of $30-80 \mathrm{~nm}$ of the islands observed in the STM images is larger than the coherence length of RHEED electrons. It explains why the RHEED did not detect the 3D-like growth of RE layers.

The magnetic measurements showed that all the samples, except samples $\mathrm{r} 4$ and $\mathrm{r} 7$, had a clear easy plane magnetic anisotropy, which is seen in Fig. 1b for $\mathrm{r} 6$ sample as an example. In the sample $\mathrm{r} 7$ the parallel and perpendicular magnetic loops are both tilted and close to each other, which suggests that the magnetization lies in between the two measured directions. In the sample $\mathrm{r} 4$ the out-of-plane anisotropy is seen (see Fig. 2b).

The models proposed by Bruno et al. [3,5] were applied to estimate the possible influence of the roughness on the magnetocrystalline surface anisotropy and the dipolar anisotropy of RE films. In the ideal flat film, when the magnetization is perpendicular to the surface, the "magnetic charges" appear on the film sides giving the high demagnetization field. In the rough film with the islands and terraces, some "magnetic charges" appear at the ends of terraces for in-plane magnetization. This results in the in-plane demagnetization field. The corresponding energy is an effective surface anisotropy of dipolar origin. Apart from the usual dipolar anisotropy

$$
E_{\mathrm{d}}=-V 2 \pi M^{2} \sin ^{2} \theta
$$

where $V$ - volume of the film, $\theta$ - the angle between the normal to the film surface and the magnetization direction, $M$ - magnetization, Bruno [5] proposed the additional positive term representing the dipolar surface anisotropy

$$
\Delta E_{\mathrm{d}}=2 S 2 \pi M^{2} \frac{3 \Delta h}{4}\left[1-f\left(2 \pi \frac{\Delta h}{\Delta l}\right)\right] \sin ^{2} \theta,
$$

where $\Delta h, \Delta l-$ the average height and diameter of the islands, respectively, $S$ - area of the film, $f(x) \in\langle 0,1\rangle$ - the function of $\Delta h$ and $\Delta l$ (see Ref. [5]).

Putting the values of $\Delta h$ and $\Delta l$ taken from Table to Eq. (2), we have found that for the samples $\mathrm{r} 3$ and $\mathrm{r} 4$ the positive term $\Delta E_{\mathrm{d}}$ is about $15 \%$ of usual dipolar anisotropy $E_{\mathrm{d}}$. Therefore it is too small to induce the perpendicular anisotropy. The magnetocrystalline surface anisotropy arises from the asymmetric environment of surface atoms. In the rough film this effect is reduced due to lower asymmetry at the edges of the terraces. According to Bruno et al. [3] the roughness modifies the magnetocrystalline surface anisotropy by an amount

$$
\frac{\Delta K_{\mathrm{s}}}{K_{\mathrm{s}}}=-\frac{2 \Delta h}{\Delta l}
$$

where $\Delta K_{\mathrm{s}}$ - reduction of the magnetocrystalline surface anisotropy energy in the rough film, $K_{\mathrm{s}}$ - magnetocrystalline surface anisotropy in the ideal flat film. The ratio $\Delta K_{\mathrm{s}} / K_{\mathrm{s}}$ was found to be less than 0.1 for all $2 \mathrm{~nm}$ and $4 \mathrm{~nm}$ thick RE layers (for thick RE layers this effect is negligible).

The different direction of magnetic anisotropy for the samples $\mathrm{r} 3$ $\mathrm{Y} / \mathrm{Gd} 2 \mathrm{~nm} / \mathrm{Y}$ (easy plane) and $\mathrm{r} 4 \mathrm{~W} / \mathrm{Gd} 2 \mathrm{~nm} / \mathrm{W}$ (out of plane) indicates however a very important role of the buffer layer type (W or $\mathrm{Y}$ ). The $14 \%$ lattice misfit 
in the case of W/RE interface induces a high magnetoelastic surface anisotropy (high lattice stresses), which is not present for $\mathrm{Y} / \mathrm{RE}$ interface (lattice misfit less than $1 \%$ ).

\section{Conclusions}

The magnetic surface anisotropy of dipolar, magnetocrystalline, and magnetoelastic origin has been studied in ultrathin RE layers. In the all investigated $Y / R E / Y$ layers we have found the easy plane magnetic anisotropy. It suggests that the presence of the dipolar and magnetocrystalline surface anisotropies only is not sufficient to overcome the usual dipolar anisotropy favoring the in-plane magnetization. On the other hand, in the W/RE/W layers the presence of the $\mathrm{W} / \mathrm{RE}$ lattice misfit, resulting in the important stresses, induces the additional anisotropy term: the magnetoelastic one. We believe that it is the main reason for the appearance of the perpendicular anisotropy observed in $W / G d 2 \mathrm{~nm} / W$ film.

According to the surface analysis in both $W / R E / W$ and $Y / R E / Y^{\prime}$ cases the roughness of investigated thin films does not change sufficiently the dipolar and magnetocrystalline surface anisotropies to introduce the reorientation of the anisotropy direction.

\section{Acknowledgments}

We would like to thank P. Aleszkiewicz for the technical support. This work was supported by the Committee for Scientific Research (Poland) under grant no. $8 \mathrm{~T} 11 \mathrm{~B} 05309$.

\section{References}

[1] W.J.M. de Jonge, P.J.H. Bloemen, F.J.A. den Broeder, in: Ultrathin Magnetic Structures, Vol. 1, Eds. J.A.C. Bland, B. Heinrich, Springer-Verlag, Berlin 1994.

[2] F.J.A. den Broeder, W. Hoving, P.J.H. Bloemen, J. Magn. Magn. Mater. 93, 562 (1991).

[3] P. Bruno, J.-P. Renard, Appl. Phys. A 49, 499 (1989).

[4] Y. Yafet, E.M. Gyorgy, L.R. Walker, J. Appl. Phys. 60, 4236 (1986).

[5] P. Bruno, J. Appl. Phys. 64, 3153 (1988). 